\title{
Das Eczem des äusseren Ohres
} von

\author{
Dr. Heinrich Anspitz, \\ em. ersten Assistenzarzte der Klinik für Hautkranke in Wien.
}

Das Eczem des äusseren Ohres ist eine so bäuffg vorkommende Localisation des Eczem's überhaupt, dass seine Besprechung manchem Otiater nicht unwillkommen sein diurfte. Bei dem reichlichen Materiale, welches mir an der hiesigen Klinik zu Gebote stand, konnte ich leicht erproben, wie sebr die Principien meines Lehrers Hebra auch in dieser Richtang stichhaltig und geeignet sind, die Theorie und Heilung dieser von den Ohrenärzten, wie ich glaube, allzu sehr gefürchteten Krankheitsform zu vereinfachen und zu erleichtern.

Es dürfte nicht überflüssig erscheinen, früher in Kürze die Ausbreitung und Beschaffenheit der allgemeinen Decke an den einzelnen Theilen des äusseren Ohres zu berühren.

Dieselbe setzt sich von allen Seiten her gleichmässig über die Ohrmuschel fort und kleidet auch den äusseren Gehörgang vollständig aus, so dass sie auch die äussere Seite des Trommelfelles überzieht.

Ueber jenen Theilen des äusseren Ohres, welche eine knorpelige, (fibröse) Unterlage besitzen, ist jedoch ihr Dickendurchmesser bedeutend geringer, weil dort das subcutane Bindegewebe und seine Fettzellen geschrumpft, and die Lederhaut durch dichtere fibröse Stränge mit dem Knorpel unmittelbar verbunden ist. 
Das Ohrläppchen dagegen besitzt eine ganz ansehnliche Schicht von Fettzellen in seinem mächtigen subcutanen Bindegewebslager.

An der concaven Seite der Ohrmuschel adhärirt die Haut dem Knorpel fester, als an der convexen, und ist über den Leisten der ersteren schwer verschiebbar.

Beim Uebergange vom knorpeligen $\mathrm{znm}$ knöchernen Theil des äusseren Gehörganges verdiunnt sie sich noch zusehends und verschmilzt dort mit dem Periost. Sie stellt an dieser Stelle und über dem Trommelfell eine zwischen der äusseren Haut und einer Schleimhaut ihrer Beschaffenheit und ihren pathologischen Veränderungen nach in der Mitte stehende Membran dar.

Ihre Gefässe und Nerven verhalten sich ganz so wie an den übrigen Theilen der allgemeinen Decke. Anders verhält es sich aber mit ihren drüsigen Apparaten.

Die Talgdrïsen sind in dor Haut der concaven Seite der Ohrmuschel, insbesondere an der Concha und der Fossa scaphoidea am zahlreichsten und grössten, so dass sie hier nach Kölliker den Durchmesser von $1 / 4$ bis $1^{\prime \prime \prime}$ erreichen und sich, was ihr Volum betrifft, rollkommen an die schönen Rosetten des mons Veneris, der labia majora und des Scrotums anschliessen.

Auch im knorpeligen Theile des äusseren Gehörganges finden sich noch Härchen und ziemlich viele Talgfollikel.

Die Schweissdriisen sind an der convexen Seite der Ohrmuschel wenig zahlreich; ihre Knäuel haben nur einen Durchmesser von 1/16 bis $1 / 12 "$, während sie in der Achselhöhle bis zu $11 / 2 "$ Dicke und 1 bis $3^{\prime \prime \prime}$ Breite erreichen, and gehören somit zu den kleinsten der ganzen Hautoberfläche.

Im knorpeligen Gehörgange vertreten deren Stelle die ganz ähnlich gebildeten Ohrenschmalzdrüsen, welche, zwischen der Haut und dem Knorpel im Unterhaut-Bindegewebe liegend, eine continuirliche gelbbraune Schichte darstellen.

Am dichtesten sind sie an der inneren Hälfte des meatus cartilagineus angehäuft und nehmen gegen dessen äussere Grenze hin allmälig an Masse und Dichtigkeit ab.

Der Meatus osseus entbehrt aller Driisen vollständig.

Nach diesen einleitenden Bemerkungen gehe ich sofort zur Schilderung des Eczem's des äusseren Ohres über.

Dasselbe trägt im Allgemeinen in dieser Localisation denselben Charakter, wie das Eczem an anderen Körperstellen. Indessen findet man leicht gewisse Unterschiede des Verlaufes heraus, wie denn auch 
die meisten Dermatologen das Eczem überhaupt auch vom topographischen Standpunkte abzuhandeln pflegen.

Das Eczem des äusseren Ohres tritt selten allein auf, d. h. ohne mit Eczemen nahe liegender Hautpartien vergesellschaftet zu sein. Unter diesen letzteren sind am häufigsten das Eczem des Gesichtes und des behaarten Kopfes. Nicht selten kommt auch der Fall vor, dass ein Eezem des Gesichtes vom äusseren Ohre beginnt, und sich dann rasch über die ganze Physiognomie verbreitet.

Das Eczem der Ohren tritt, wie alle anderen Eczeme, entweder in acuter oder in chroniseber Form auf.

Die Kennzeichen des acuten Eczems sind:

Bedeutende Schwellung und Röthung der Haut, vollständige oder abortive Bildung von Bläschen.

Die rothe, von dem Hinterhaupte abstehende, bisweilen durch Oedem teigig anzufühlende Ohrmuschel wird durch ihre bedeutende Volumsvermehrung in schweren Fällen zu einer unförmlichen Masse. Dicht an einander gedrängte Bläschen mit serösem Inbalte ergiessen beim Platzen eine reichliche, bisweilen röthlich tingirte Flüssigkeit, welche iiber das Ohrläppchen herabträufelt.

Dies ist die typische Form des vesiculösen Eczem's der Ohren. Häufig: erfolgt aber die Evolution desselben so rasch, dass es gar nicht zur Bläschenbildung kommen kann, sondern diese sofort abortiv za Grunde gehen und nur eine, massenhaftes Fluidum absondernde, stark geröthete und infiltrirte mit Excoriationen bedeckte Basis zuriicklassen. In diesen Fällen hat das Eczem in der Form des Eczema rubrum seu madidans begonnen.

Beim acuten E. fehlen die subjectiven Empfindungen von Spannung, Druck, Hitze, geringem Jucken, Ameisenlaufen, Brennen sehr selten; und wenn die Schwellung sich auch in den äusseren Gehörgang erstreckt, oder es auch hier zur Bläschenbildung gekommen ist, gesellt sich sogar bisweilen Schwerhörigkeit durch Verstopfung hinzu.

Alle diese Erscheinungen schwinden in der Regel nach kurzer Zeit, in wenigen Stunden oder Tagen - aber sie recidiviren ofter.

Treten solche Rückfälle mehrere Male ein, oder weicht die oben geschilderte Form des primären acuten Eczem's nur, um einer anderen Symptomengruppe Platz zu machen - so ist das acute E. nach und nach in ein chronisches übergegangen.

Seinem Wesen nach ist dieses nicht von ersterem unterschieden, sondern bildet eine Fortsetzung desselben. Man kann daher auch mit gotem Recht sagen, das chronische Eczem beginne in Form des E. vesiculosum oder rubrum. 
Wenn man Kranke mit Eczemen der Ohren zu Gesichte bekonmt, so wird man jedoch in vielen Fällen weder das Bild des BläschenEczems noch jenes des rothen nässenden wahrnehmen, sondern viel häufiger jene Form, welche als $E$. impetiginosum (Impetigo) bezeichnet wird.

Es gerinnt nämlich die aus den Bläschen oder aus ihrer Basis bei abortivem Verlauf sich massenhaft ergiessende Flüssigkeit mit ihrem reichlichen Eiweissgehalte sehr schnell and verwandelt sich zu gelben oder gelbbraunen auf excorïrtem Grunde sitzenden Borken. Derselbe Vorgang findet in der Regel gleichzeitig im Gesichte statt, und stellt so das als Crusta lacea, von Alibert als Melitagra flavescens bezeichnete Krankheitsbild dar. Dieses bei Kindern sehr häufige, aber auch bei Erwachsenen nicht seltene Uebel wird vom Publikum und von vielen Aerzten noch sehr respektirt, und die Borken geradezu als noli me tangere behandelt.

Ich hatte gerade in jüngster Zeit Gelegenheit, mehrere Fälle von Milchschorf bei Kindern zu sehen, die man aus Vorurtheil nicht hatte behandeln lassen wollen, aber nach kurzer Zeit dazu gezwungen war, als sich aus den Gesichts-Eczemen universelle E, herausgebildet hatten.

Die Borken des E. impetiginosum am Ohre sind bisweilen so umfangreich, dass sie das ganze äussere Ohr bedecken und wie Zapfen von demselben herabhängen, so dass sie Alibert als dartre stalaictiforme, Tropfsteinflechte, bezeichnete.

Die letzte typische Formveränderung, welche das Eczem eingehen kann, ist endlich jene in das schroppige Eczem (E. squamosum, Pityriasis rubra), das eigentliche chronische Eczem. Schon der Name drückt aus, dass man es hier mit einer reichlichen Epidermis-Entwicklung zu thun habe, welche über einem rotben Grunde fort und fort ibren Verlauf nimmt, während hie und da noch recrudescirende nässende Stellen auftauchen.

Ausser den bisher geschilderten Erscheinungsarten des Eczems tritt indessen am Ohre nicht selten ein partielles $\mathrm{E}$. auch in der Weise auf, dass entweder in der Concha, besonders häufig aber an der hinteren Fläche der Ohrmuschel nahe ihrer Ansatzstelle vor dem processus mastoideus anstatt kleinerer Excoriationen tiefere spaltförmige Schrunden (Rhagaden) sich zeigen, über welchen die Epidermis verloren gegangen ist, und zwischen deren rothen Rändern gewöhnlich reichliches Fluidum hervorquillt.

Diese, besonders im Ohr sehr häufige Form des Eczems gehört dem $E$. rubrum an und wird von vielen, besonders französischen 
Autoren als Intertrigo (I. secrétant von Devergie) beschrieben. Diess ist um so weniger gerechtfertigt, da gerade letzterer Schriftsteller als eine eigene Form das E. fendillé annimmt, welches er aber auf die Ober - und Unterschenkel und die Vorderarme bezüglich ihres Vorkommens beschränkt.

Es erübrigen nunmehr noch zwei Eczemformen, deren wir nicht Erwähnung gethan haben, das papulöse und pustulöse E. In der That kommen aber diese beiden nur sebr selten am äusseren Ohre vor.

Bloss am Ohrläppchen hatte ich bisher öfter Gelegenheit, das Entstehen von Knötchen mit gleich darauf folgender Schuppenbildung (E. papulosum, lichenodes), bei gleichzeitiger Bläschenbildung in der Ohrmuschel zu beobachten.

Am seltensten kommt es zur Entwickling dex pustulösen Form, was bei der massenhaften Flüssigkeit, die sich in den Bläschen entwickelt und ausgeschieden wird ind bei der dadurch schnell bewirkten Krustenbildung leicht begreiflich ist. Nur langsam sich entwickelnde Bläschen mit widerstandsfähiger Epidermis lassen in der Regel die Entstehung von Pusteln zu.

Wenn das Eczem das ganze Ohr ergriffen hat, kommen im Verlauf des Processes oft alle diese Formen neben einander vor: Am Ohrläppchen Papeln oder Bläschen; an der hinteren Seite der Ohrmuschel Rhagaden; an der concaven Fläche der Muschel und im äusseren Gehörgange Schwellung, Röthe, Nässen, Borkenbildung; endlich bei noch vorhandener starker Schwellung und Verstopfung des äusseren Gehörganges mit Sehwerhörigkeit - im äusseren Ohre schon die Form des in der Heilung befindlichen squamösen Eczems,

Fassen wir nun die charakteristischen Merkmale, wodurch sich das Eczem des äusseren Ohres vor anderen Eczemen auszeichnet, zusammen, so finden wir als solche:

1) Das sonst seltene Auftreten von Schwellung hohen Grades.

2) Die massenhafte Absonderung seröser Flüssigkeit.

3) Das häufige Auftreten von Rhagaden.

4) Die Obturation des äusseren Gehörganges und die Schwerhörigkeit.

Aetiologie und Vorkommen.

Aus der schon angegebenen Gleichzeitigkeit des Vorkommens mit den Eczemen des Gesichtes und des behaarten Kopfes ergibt sich, 
dass die ätiologischen Momente annähernd dieselben sein werden, wie bei jenen. Ich unterlasse daher eine detaillirte Aufzählung derselben und erwähne nur, dass sowohl äussere Reize (Aetzmittel, Bäder, Kälte, Hitze u. s. w.) als innere Zustände (Scrofulose, Rhachitismus, Chlorose, Hysterie u. dgl.) mit der Entstehung von Eczem der Ohren in Connex gebracht werden, Die meisten Schriftsteller geben übrigens an, dass dasselbe bei Kindern in der Lactationsperiode, dann bei Mädchen mit Menstruations-Anomalien, ferner zur Zeit des Mannbarwerdens derselben und ebenso beim Eintritt der climacterischen Jahre besonders häufig vorkomme.

Wenn Devergie jedoch behauptet, das Eczem der Ohren sei sehr gewöhnlich beim Weibe und sehr selten bcim Manne, so ist dies nicht ganz richtig. Sowohl bei Kindern als bei Erwachsenen männlichen Geschlechtes sieht man solche Eczeme hänfig genug; nur freilich weniger häufig, als beim weiblichen Geschlechte. In den meisten Fällen sind beide Ohren gleichzeitig erkrankt.

\section{Diagnose.}

Sowohl die charakteristische Form und der Verlauf, als die Miterkrankung des Gesichtes oder behaarten Kopfes machen die Diagnose in der Regel nicht sehr schwierig. Am äusseren Ohre ist aber eine Erkrankung des Drüsenapparats nicht selțen, welche zu Verwechslung mit Eczem Anlass geben kann, die Seborrhoe (Aene sebacea nach Biett). Dieselbe beruht bekanntlich auf stärkerer Absonderung von Hauttalg durch die Talgfollikel, und kennzeichnet sich durch einen fettigen Glanz der Haut in der Umgebung der bisweilen zu kleinen Knötchen geschwellten Talgdrüsen und durch Bildung von weisslichen, nach und nach dunkler werdenden und vertrocknenden Schïppchen, die sich, zwischen den Fingern gerieben, ölig anfühlen. Die Seborthoe kann mit oder ohne Congestion der Haut auftreten; niemals aber kommt es dabei zu Infiltration.

Das physiologische Prototyp der Seborrhoe bildet die sogenannte käsige Schmiere der Neugebornen, welche gerade die Ohrmuschel und die Gegend des Warzenfortsatzes mit einer besonders dicken Schichte zu überziehen plegt. Das auffallendste pathologische Beispiel von Seborrhoe findet sich gewöhnlich an der Nase, welcher bisweilen in Folge mangelhafter Reinigung so dicke Sebum-Massen aufgelagert sind, dass sie das Volum der Nase um das 2-3fache vermehren können. Nach jahrelangem Bestande genügt dann oft die einfache Ab- 
hebung derselben und Reinigung der darunter liegenden gerötheten aber nicht excoriirten Haut zur Heilung des Uebels.

Am äusseren $\mathrm{Ohr}$ findet sich die Seborrhoe natürlich nur dort, wo sich Talgdrüsen befinden, also weniger an der convexen, als an der concaven mit sehr grossen Talgfollikeln versehenen Fläche der Ohrmuschel, deren Vertiefungen durch die reichlichen Schüppchen bei mangelnder Reinigung fast ausgeglichen werden können.

Was den äusseren Gehörgang betrifft, so sind in seinem knorpeligen Theile sowohl die Talgdrüsen, als die Ohrenschmalzdrüsen, welche die Stelle der Schweissdrüsen vertreten, reichlich vorhanden. Die so häufig vorkommende und beschriebene Anhäufung von Ohrenschmalz mit Verstopfung des Gehörgangs und Schwerhörigkeit oder gar Taubheit ist, wie ich glaube, weniger den Ohrenschmalz- als den Talgdrüsen in die Schuhe zu schieben. Das Secret der Ohrenschmalzdrüsen allein konnte man bisher nicht untersuchen; nun sind aber dieselben den Schweissdrüsen ganz conform gebaut. Wenn man nun bedenkt, dass im knorpeligen Gehörgang viele und grosse Talgfollikel sitzen, so wird man vielleicht geneigt sein, die Urheberschaft der Ohrenschmalzkrankheit nur in so fern den Ohrenschmalz- oder besser Ohrenschweissdrüsen zu vindiciren, dass ihr reichliches Secret die mechanische Möglichkeit der Verstopfung herbeifuhrt. Ein grosser Antheil an der Bildung des eigentlichen Caerumen möchte hingegen den Talgdrüsen zuzuerkennen sein. Es ist bekannt, dass in den Ohrenschmalz-Pfröpfen sich öfter runde oder ovale Körperchen finden, die nach aussen deutliche, concentrisch angeordnete Epithelzellen, nach innen nur Fett und moleculare Masse zeigen. Diese Körperchen wurden von Einigen als das Produkt massenhafter Secretion der Obrenschmalzdrüsen bei plötzlich eintretender oberflächlicher Entzündung des Gehörganges angesehen - eine Erklärung, welche Schwartze in seinen jüngst erschienenen Beiträgen für unzulässig crklärt. Ich stimme dem Letzteren vollkommen bei, und glaube, dass die Talgdrüsenpfröpfe an der äusseren Haut die passendste Analogie für jene Körperchen bilden, welche durch den allseitig stattfindenden Druck des angesammelten Caerumen's im Gehörgange eine Schicht der EpithelAuskleidung der Haarbälge oder Talgdrüsen-Ausführungsgänge mitgerissen haben.

Die Seborrhoe des äusseren Ohres unterscheidet sich nach dem früher Gesagten vom Eczema rubrum durch die fehlende Anschwellung sowohl der Ohrmuschel als der Haut des äusseren Gehörganges, durch das Fehlen der Infiltration, des Nässens, durch das fettige Anfühlen, das geringe Jucken. Das Eczema squamosum, welches noch 
am ehesten damit verwechselt werden könnte, ist einerseits durch die vorher gegangenen Symptome des E. rubrum, andrerseits durch das stellenweise Vorkommen von Excoriation und durch die immer noch starke Infiltration der Haut auffallend genug von der Seborrhoe unterschieden.

Die Seborrhoe des äusseren Olhres kommt häufig mit der gleichen Erkrankung des behaarten Kopfes vor, eben so oft aber gesellt sich zu Seborrhoe des letzteren Eczem der Ohren, welches schwindet, wenn die Sebum-Absonderung des Kopfes sich vermindert.

Wir haben noch die Differential-Diagnose des Eczem's von der Psoriasis, dem Erisypel, der Entzündung des äusseren Gehörgangs und dem Catarrh desselben zu besprechen.

Von der Psoriasis mit ihren getrennt stehenden, von dichten weissen Schuppen bedeckten, leicht blutenden Plaques ist das squamöse Eczem der Ohren in der Regel leicht zu trennen. Einzelne Uebergangsformen zwischen beiden Krankheiten, die an anderen Körperstellen bisweilen die Diagnose schwierig machen, sind wohl am äusseren Obre nicht wesentlich hinderlich, weil sich die Frage in der Regrel an einem geeigneteren Territorium, dem behaarten Kopfe z. B. schon früher entschieden hat.

Der Verlauf des Erysipels ist ein so charakteristischer, dass ich auf diese Krankheitsform, welche mit dem Eczem nur die Schwellung und Röthe gemein hat, nicht näher eingchen will. Eben so wenig auf die acute und chronische Entzündung des äusseren Gelörganges, welche bei dem Fehlen gerade der für das Eczem wesentlichen Symptome nur durch völlige Unkenntniss der letzteren mit demselben zusammengeworfen werden konnten.

Was endlich den Catarrh des äusseren Gehörganges betrifft, so ist dessen Hauptsitz und zugleich die Ursache des eitrigen Ohrenflusses der tiefere Theil des Meatus auditorius, dessen Ueberzug in einer den Schleimhäuten ähnlichen Weise erkrankt. Die secernirte Flüssigkeit ist von dem Secret des Eczems leicht zu unterscheiden. Sowohl das Eczem als die Seborrhoe können übrigens von Catarrh des äussern Gehörganges begleitet oder gefolgt sein.

\section{Prognose und Verlauf.}

Das acute Eczem des Ohres geht in kurzer Zeit vorüber, aber es macht öfter Recidiven. Das chronische E. gehört dagegen zu den hartnäckigsten Formen dieser Erkrankung und hält in dieser Beziehung gleichen Schritt mit den behaarten Theilen des Körpers. 
Doch wird es nicht viele Fälle geben, an denen man gänzlich verzweifeln müiste, wenn nur die entsprechende Behandlung eingeleitet wird. Entwedar es hört die früher constant wiederkehrende Gelegenheits-Ursache (Dyspepsien, Schwangerschaften u. s. w.) mit der Zeit auf, oder die Recidiven erschöpfen sich von selbst, oder cin oder das andere Mittel übt plötzlich einen überraschenden Erfolg.

\section{Therapie.}

Die früher herrschende Scheu vor der Heilung von Eczemen des Ohres dürfte gegenwärtig bei gebildeten Aerzten seltener geworden sein. In der grossen Zahl von Fällen von Gesichts-, Kopf- und OhrenEczem, die ich beobachtete, kam nie irgend eine Metastase zur Behandlung. Die oft gehörte Behauptung, das Eczom „schlage sich, wenn es vom Hus*eren Ohre vertriebrn werde, auf das Gehör", beruht, wie Hebra vichtige bemrrkt, auf der nurichtigen Deutung des Faktums, dass oft cret im Verlauf der Erkrankung die tieferen Partien des äu-sern Geliörgangr's daron ergriffen werden, wodureh es zur Oturrhoe oder zur Gichörstörung konmen kann.

lch muss zu örderst bemtrhen, fhass die an liänfigstrn angewendeten inneren Mittl, die Solventia unl blutreinigenden Decocte auf das Eczem sellust heinen Enflus ïlen. Frcilich soll danit nicht geläugnet werden, dass z. B. melrtäyige Obstruction der Gedärme das Allgemeinbefinden und daher auch den Zustand eines Eczems zu verschlimmern vermögen.

Von allen anderen, in Gebrauch gezogenen inneren Mitteln kann nur dem Arsenito (in. Form der Fowler'schen oder Pearson'schen Lösung, oder der asiatischen Pillen) eine Einwirkung auf länger bestehende Eczeme zugesprochen werden. Einerseits aber liegt es in der Natur dieses Mittels, dass man nicht gern davon Gebrauch macht, andrerseits ist sejne Wirkung nicht so eclatant, um nicht durch andere äussere Mittel vollständig ersetzt werden zu können.

Von Blutentziehungen habe ich noch keinen. Nutzen gesehen. Uebrigens mag der Praktiker immerhin bei einem acuten Eezem mit starker Schwellung und Röthung, um sein Gewissen zu erleichtern, einige Blutegel, aber recht weit von der eczematösen Hautpartie entfernt, hinter die Ohren appliziren.

Die Behandlung des acuten Eczems ist überhaupt eine wenig complizirte. Die Hauptregel dabei ist: Man thue so wenig als mög- 
lich. In der Mehrzahl der Fälle werden auch Salben, Pflaster u. s. w. gar nicht vertragen.

Man beschränke sich also zunächst darauf, die erkrankte Stelle gegen die Luft zu schützen. Ich bin überzengt, dass eine grosse Zahl von Eczemen einfach durch den Abschluss der Atmosphäre geheilt werden könnte. Dass ein Gypsverband ein ausgebreitetes Eczem des Unterschenkels vollständig heilte, sah ich selbst in einem Falle.

Zu diesem Zwecke leistet beim acuten Eczem die besten Dienste ein aus Roggen - oder Reismehl bestehendes Streupulver, dem man noch Flores zinci, Taufstein, Federweiss (pulv. alumin. plumosi) u. dgl. m. zusetzen kann. Z. B.

Rp. Flor. zinci drachmas duas

Pulv. alum. plum.

Amyli puri ana unciam

M. d. s. Streupulver.

Dieses Pulver wird mittelst eines Feder- oder Charpiepinsels so oft als möglich auf die eczematösen Stellen aufgetragen, und bewirkt in der Regel Abnahme des Juckens und Brennens. Hie und da wird es dagegen auf excorïrten Stellen nicht vertragen; dann greift man einerseits zu kalten Ueberschlägen, andrerseits zu leichten Adstringentien, als Sulfas zinci, Plumbum aceticum n. s. w. in Lösung.

Unter dieser einfachen Behandlung wird das acute Eczem des Ohres schr häufig in kurzer Zeit schwinden.

Eine complicirtere Medication erfordert das chronische Eczem. Ich erwähne zunächst des Wassers und zwar in Form der Douche. Bei hartnäckigen chronischen Eczemen ist die kalte Regendouche, von einer mässigen Höhe auf die Ohrmuschel geleitet und mehrere Male des Tages angewendet, von grossem Einfluss auf die Verminderung: der Reizempfindung in der Haut, und oft auch wäbrend einer anderen Behandlungsweise gar nicht zu entbehren.

Von kalten und lauwarmen Ueberschlägen, ferner solchen mit Malve, Althaea u. s. w. habe ich bei chronischen Eczemen nur wenig Erfolg gesehen.

Wenn man die Douchekur nicht allein oder gar nicht anwenden will, so ist zunächst das Augenmerk darauf zu richten, dass die Haut für die Einwirkung von Arzneimitteln in geeigneten Zustand versetzt werde. Es müssen daher die beim E. des Ohres gewöhnlich so massenhaften Ausscheidungen (Impetigoborken) weggeschafft werden. Diese Grund-Operation, welche bei Kindern und Erwachsenen in gleicher Weise stattzufinden hat, wird am Besten durch ölige Substanzen bewirkt. Es ist vollkommen gleichgültig, welche Art von Oel oder Fett 
man anwendet, aber eine wichtige Bedingung ist die, dass dasselbe durch längere Zeit ohne Unterbrechung fest mit der Hotit in Berührung gehalten werde.

Da es an der Ohrmuschel schwierig ist, mit flüssigem Fett zu manipuliren, applicirt man hier am Besten einofache Salbe (Unguentum simplex, Crême celeste, Cold cream u. dgl.j. Dicselbe wird messerrückendick auf einen Leinwandfleck aufgetragen, der nach vorne und rückwärts um den als Hypomochlion dienenden Helix umgebogen und oben und unten durch Bändehen an den Ansatzstellen des Ohres befestigt wird.

Hiedurch ist die convexe Seite der Ohrmuschel, das Ohrläppchen Helix, Antihelix, Fossa scaphoidea und Tragus vollständig und glatt bedeckt. Die Concha, der Antitragus und die Fossa triangularis mïssen dagegen einfach mit der Salbe bestrichen und dann mit Baumwolle bedeckt werden, welche durch den darüber gespannten Leinenfleck in der Ohrmuschel festgehalten wird.

Den äussern Gehörgang wird man am besten mit flüssigem Oel (Olivenöl, Mandelöl, aber nicht Leberthran, der leicht ranzig wird) und zwar einige Male im Tage anfüllen und mit Baumwolle dessen Ausfliessen verhindern.

Oft gelingt es auf diesem Wege ohne Anwendung anderer Mittel das Eczem nach längerer oder kürzerer Zeit zu heilen. Zugleich kann man hiebei jede Berufsstörung des Kranken völlig vermeiden. Unter allen hieher gehörigen Salben ist am meisten das von Hebra in die Praxis eingeführte Ung. Diachyli zu empfehlen, dessen Formel nach Steinhüuser lautet:

Rp. Ol. olivar. optimi une. 15

Lithargyri unc. 6 et 36

Coq. l. a. in ung. molle

dein adde

OI. lavandul. $3 \mathrm{ij}$.

M. f. ung.

In kleinerer Quantität kann man dasselbe aber auch unmittelbar aus dem Diachylonpflaster bereiten lassen:

Rp. Empl. Diachyli simpl. liquefacti

Ol. olivar. ana unciam

M. f. lege artis ung. molle.

Von vielen Aerzten werden beim Eczem Zinksalben als besonders wirksam empfohlen, und sind in der That, wenn nach der angegebenen Methode angewendet, sehr brauchbar. Erasmus Wilson empfichlt 
besonders das Ung. oxyd. zinci benzoinatum mit Spiritus vini $(8: 1)$. Die Vorschrift desselben lautet:

Rp. Adipis une. sex.

Gummi Benzoini pulveris. drachm.

Liquef. leni calore per 24 horas in vase clauso, dein cola et adde

Oxydi zinci purif. unciam

Misce et exprime per linteum.

Die Anwendung der bisher erwähnten Mittel wird nach dem Gesagten bei der Form des Eczema impetiginosum und rubrum an vortheilhaftesten sein. Wenn aber das E. gleich ursprünglich als schuppiges E. aufgetreten oder nach und nach zu einem solchen geworden ist, dann kommt man gewöhnlich mit erweichenden Mitteln allein nicht zum Ziele.

In solchen Fällen ist die Anwendung leicht ätzender Mittel oder der Theerpräparate angezeigt.

$\mathrm{Zu}$ den ersteren gehören: Lösungen von Arg. nitricum, von $S u$ blimat, Zinkoxyd, Kali causticum (2 bis 3 Gran auf die Unze), oder Salben insbesondere mit weissem Quecksilber-Praecipitat (1 Drachme auf 1 Unze).

Weitaus vorzuziehen sind aber nach meiner Erfahrung die Theerpraparate und zwar: das Oleum fagi, musci oder cadinum (zur Hälfte mit Alkohol oder Oel gemischt). Diese werden zwei Mal täglich mittelst eines Borstenpinsels in dünner Schicht auf die Haut aufgetragen, und dann ein Streupulver darüber gestreut. Nur wenn der Geruch des Theers nicht vertragen wird, wende man die weniger penetrant riechende Carbolsäure, ein farbloses, in Alkobol und siedendem Wasser lösliches Destillationsprodukt des Theers in folgender Form an:

Rp. Acidi carbol. drchm, 1 bis 2

Glycerini et

Alcoholis ana unc. semis

Aq. destill, unc. sex.

Die Theerseife (von der Société hygiéinique in Paris gut bereitet) ist bei der für die Behandlung ungünstigen Conformation des äusseren Ohres wohl nicht leicht anzuwenden. Oefter ereignet es sich, dass der Theer die Röthung der Haut vermehrt oder bei längerem Gebrauch zur sog. Theer-Acne führt.

Hebra wendet daher seit einiger Zeit, insbesondere bei Kindern, gern zugleich mit dem Theer erweichende Salben an, welche sogleich nach dem Eintheeren applicirt und vor der neuerlichen Einpinslung durch Seife abgewaschen werden. 
Von der Schmierseife oder ihren Lösungen (Kalicrême, Spir. saponatus kalinus) mache ich bei Eczem der Obren nur Gebrauch, um die Haut täglich einmal durch Abreiben vermittelst eines Flanellstückes von der Salbe oder dem Theer zu reinigen. Methodisch als selbstständiges Heilmittel (z. B. in Form von Umschlägen, welche dann immer ätzend wirken,) die Seife anzuwenden, fand ich bisher beim Eczem der Ohren nicht für praktisch.

Von den Schuefelpröparaten habe ich ebenfalls nicht leicht einen günstigen Erfolg, sondern eher Zunahme der Eczem-Erscheinungen gesehen.

Das Linimentum calcis (Rau), Jodlcalium (Valleroux) und Traumaticin (Wilde) habe ich nie versucht, glaube aber a priori an ihrer Nützlichkeit zweifeln zu müssen.

Zum Schluss muss bemerkt werden, dass sich diese Behandlungsweise des Ohres je nach der Art der am Kopf oder Gesichte bei gleichzeitigem Eczem dieser Theile eingeschlagenen Methode nothwendig modificiren wird, damit nicht allzu viele verschiedene Mittel zu gleicher Zeit angewendet werden.

Für den äussern Gehörgang genügt die oben erwähnte Oeleinträuflung vollständig, wenn nur zugleich das Eczem der Ohrmuschel zum Schwinden gebracht wird. 